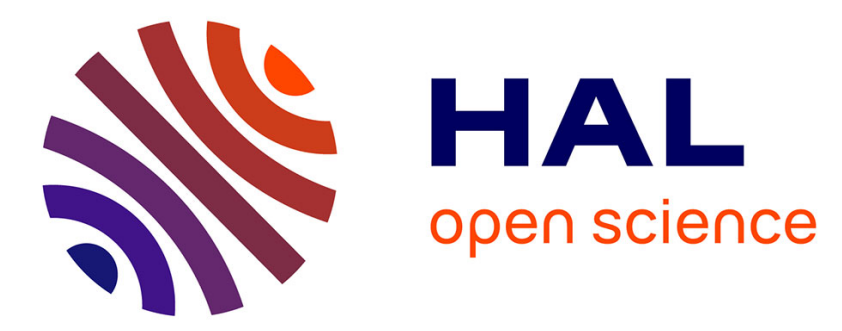

\title{
Some observations on the impact of a low solubility ionic solution on drying characteristics of a model porous medium
}

\author{
Mamadou Diaga Seck, Emmanuel Keita, Philippe Coussot
}

\section{To cite this version:}

Mamadou Diaga Seck, Emmanuel Keita, Philippe Coussot. Some observations on the impact of a low solubility ionic solution on drying characteristics of a model porous medium. Transport in Porous Media, 2019, 128 (3), pp.915-928. 10.1007/s11242-018-1169-0 . hal-02912596

\section{HAL Id: hal-02912596 \\ https: / hal-enpc.archives-ouvertes.fr/hal-02912596}

Submitted on 6 Aug 2020

HAL is a multi-disciplinary open access archive for the deposit and dissemination of scientific research documents, whether they are published or not. The documents may come from teaching and research institutions in France or abroad, or from public or private research centers.
L'archive ouverte pluridisciplinaire HAL, est destinée au dépôt et à la diffusion de documents scientifiques de niveau recherche, publiés ou non, émanant des établissements d'enseignement et de recherche français ou étrangers, des laboratoires publics ou privés. 


\title{
Some observations on the impact of a low solubility ionic solution on drying characteristics of a model porous medium
}

\author{
M. D. Seck*, E. Keita, P. Coussot \\ Université Paris-Est, Laboratoire Navier (ENPC-IFSTTAR-CNRS), 2 Allée Kepler, Champs sur \\ Marne, France \\ * Present Address: Marley Eternit, Lichfield Road, Branston, Burton upon Trent, DE14 \\ 3HD, UK
}

\begin{abstract}
We study the impact on the drying rate, of the presence of suspended elements, such as calcium sulfate ions with a low solubility, in the interstitial fluid of a porous medium. In order to single out this process in the complexity of a porous medium, we study it through drying in a simple capillary exhibiting characteristics such that it reproduces some critical aspects of drying of porous media. Another specificity of our work is that we focus on the evaporation of initially ion saturated solutions. We first show that in such a capillary the drying process varies depending on the wettability characteristics. Typically the drying rate is much smaller with hydrophobic surfaces because of the airliquid interface which tends to withdraw inside the medium, while for hydrophilic surfaces there remains a continuous liquid film up to the entrance. Then it appears that an ionic solution dries slower than a pure liquid, because the crystals formed along the capillary walls tend to induce a dewetting of the capillary entrance, pushing inwards the first liquid-air interface from which most of the evaporation occurs. An experiment with a model colloidal suspension further illustrates this mechanism: the accumulation of solid particles along the wall forms a deposit which pushes inwards the first liquid-air interface from which evaporation takes place. Finally we look at the impact, on the drying characteristics, of the presence of different additives in the ionic solution.
\end{abstract}

\section{Introduction}

Salt presence and crystallization in building materials are responsible for major damages of modern structures (Liu et al. 2014) and cultural heritage (Sawdy et al. 2008). Similar effects in rocks can alter CO2 injectivity (Peysson et al 2011) and are at the origin of various geomorphological processes (Rodriguez-Navarro and Doehne 1999). For example ions dissolved during imbibition can then, during drying, reach the solubility limit and precipitate as crystals below (subflorescence) or above (efflorescence) the sample free surface. In the case of crystallization, these crystals may clog the porous structure (Espinosa-Marzal and Scherer 2013, Derluyn et al. 2013), and leading to flakings, crumblings (Kramar et al. 2010), or a reduction of the compression strength of the material (Foraboschi and Vanin 2014). The mechanisms at the origin of these damages have been discussed: crystallization pressure thanks to solid volume change from thenardite to mirabilite (Scherer 2004, Flatt 2002, Schiro et al. 2012, Flatt et al. 2014, Tsui et al. 2003) and supersaturation. It was also acknowledged that the remaining liquid films can play a significant role on crystallization processes (Espinosa-Marzal and Scherer 2010). Extensive studies (Rodriguez-Navarro et al. 1999, Schultz and Schlünder 1990) show the variety of crystallization patterns observed depending on solution properties (salt type and additives). Again, further studies on salt and damage localization (Rodriguez-Navarro et al. 1999, ShahidzadehBonn et al. 2010, Desarnaud et al. 2013) highlight that possible damages are directly linked to the localization of crystallized salts within the porous media. A better understanding of the latter requires 
a description of the salt transport dynamics, spatial distribution and crystallization, which are directly linked to the drying mechanisms.

For efflorescence the basic theoretical concepts of salt transport resulting from water flow during drying were confirmed by following the salt distribution by MRI (Pel et al. 2004, Petkovic et al. 2010). It was then shown that crystals deposited at the sample free surface behave as a porous medium which thanks to its smaller pore structure (Veran-Tissoires et al. 2012, Sghaier and Prat 2009) pumps the liquid and brings ions around the most external liquid-air interface to form new crystals. Another study showed in addition that the structure of the efflorescence layer can evolve with successive imbibitiondrying cycles, process which will successively modify the drying rate (Desarnaud et al. 2015). Microtomography observations of the three phases around the sample top provided a detailed description of the process, which makes it possible to establish a model of crust formation dynamics as a function of drying rate (Norouzi Rad et al. 2015). Finally further details on the local mechanisms of evaporation from the evolving salt crust were obtained through high-resolution thermal imaging (Shokri-Kuehni et al 2017). Besides, it was shown that some unexpected effects (in drying rate variations) can result from the interplay between crust widths and evaporation conditions (Gupta et al. 2014a, Gupta et al. 2014b). At last, it was recently shown that, in the presence of salt in solution, the increase of the fraction of hydrophobic grains in a hydrophilic-hydrophobic grain mixtures has minor impact on the drying rate (Bergstad and Shokri 2016).

For example, for plasters, after the chemical (dissolution-precipitation) hydration reactions which typically occur in less than one hour and allow to get the basic solid porous structure of the material, a significant excess of water then has to be removed from the (saturated) freshly formed porous structure. However, at the end of this hydration process (due to precipitation of dihydrates) the interstitial solution is saturated with ions $\left(\mathrm{Ca}^{2+}, \mathrm{SO}_{4}{ }^{2-}\right)$, and will precipitate as gypsum crystals and accumulate in some regions as soon as some water is extracted by evaporation. Thanks to the high dry rates we are working with, the advection is much higher than the diffusion (i.e. the Peclet number is much larger than 1), hence salts are transported towards the free surface (Sawdy et al. 2008) and then can either crystallize inside the plaster or on top of the free surface (Petkovic et al. 2007). It was also suggested that this salt crystallization leads to the formation of a structure inside the plaster, whose characteristics depend on the drying rate and in turn impacts the drying rate (Vosten and Hersfeld 1976, Seck et al. 2015). From Magnetic Resonance Imaging and X-Ray Microtomography measurements it was recently shown (Seck et al. 2016) that crystals deposit around the air-liquid interface the closest to the sample free surface, which induces an apparent recession of this interface towards the interior of the sample and the distribution of crystals deposited during evaporation, essentially depends on the history of saturation. It was then suggested that the drying dynamics results from vapor diffusion through the less porous layers of crystal accumulated below the sample free surface. This in particular makes it possible to predict the dramatic decrease of the drying rate after successive imbibition-drying cycles (Seck et al. 2016).

A more detailed view of this process would be useful to fully understand the physical effects at work. In that aim it can be instructive to use a simpler porous structure. For example, with a sintered packing of glass beads initially saturated with a low concentration ionic solution (gypsum saturated solution: $\mathrm{SO}_{4}^{2-}, \mathrm{Ca}^{2+}$, with a mass concentration $\approx 2.65 \mathrm{~g} / \mathrm{l}$ ) and submitted to a given air flux, a drop of the drying rate may be observed from the beginning of the drying process (Seck 2015), whereas with pure water filling the medium and identical air flux, the drying rate remains constant down to low saturation. Finally it requires $27 \mathrm{~h}$ to get a saturation of $20 \%$ with the ionic solution whereas it requires only $16 \mathrm{~h}$ with pure water. This confirms that ions from gypsum $\left(\mathrm{SO}_{4}^{2-}, \mathrm{Ca}^{2+}\right)$ crystallization can by itself affect the drying characteristics of a porous medium. Note that the saturation vapour pressure of water 
may be modified by the presence of ions in solution. According to the Raoult law and considering that the ion concentration in our case is very low, we expect a negligible effect (Seck 2015). However, with $\mathrm{NaCl}$ this impact was observed to be significant, inducing a decrease of the drying rate by almost 50\% (Norouzi Rad and Shokri 2012).

Furthermore more model porous structures had been used to get a detailed view of the dynamic process at the local scale. Recent observations (Rufai and Crawshaw 2017) of drying of $\mathrm{NaCl}$ solutions in a micromodel directly show that crystallization tend to blocks some paths, which decreases the drying rate. Other experiments in other types of 2D (Eloubaki et al 2011) or 3D (Hidri et al. 2013) model porous media showed that the drying rate can be affected by efflorescence mechanisms. Drying of $\mathrm{NaCl}$ salt solutions has even been studied in simple tubes: an experimental study was carry out with 1 $\mathrm{mm}$ cylindrical tubes, and the processes of crystallization and deposit were modelled (Naillon et al. 2015); the patterns of salt crystallization from droplets and in square tubes were also reviewed (Shahidzadeh-Bonn et al. 2008). The same processes, but now in tube with cross-square section which leads to considerable change due to the persistence of liquid films along the corners, was modelled (Camassel et al. 2005).

Here we focus on the drying of an ionic solution of low solubility (corresponding to that found in plasters (see above)), from capillaries exhibiting characteristics such that they reproduce some critical aspects of drying of porous media: the geometry consists in a thin channel with a rectangular section, open on one side (left hand side in the figures of this paper), so that capillary effects remain dominant during most of the drying process, as in a porous medium (Keita et al. 2016), and it is generally possible to follow the evolution of the air-liquid interface in time. Another specificity of our work is that we focus on the evaporation of initially ion saturated solutions. In this context we will review some observed patterns but we will not attempt to understand the mechanisms of crystallization or deposit at a local scale, and the origin of these patterns; instead we will mainly concentrate our attention on the impact of these phenomena on the drying rate of the whole system.

\section{Materials and methods}

We performed drying experiments with $5 \mathrm{~cm}$ long hollow tubes manufactured with borosilicate glass with a rectangular section. The internal width and thickness of the tube are respectively 0.1 and $1 \mathrm{~mm}$; the corners of the section are slightly rounded (see Fig.1). For the tests with colloidal suspensions we used the same channel type but with a $2 \mathbf{~ m m}$ width and a more imperfect rectangular cross-section (see Keita et al 2016a). Note that these channels were obtained at different periods from the same supplier and with the same reference, which suggests that an evolution of the manufacturing process is likely at the origin of this difference.

Once saturated with a liquid, the capillary is held horizontally and glued at one of its extremity with Loctite ${ }^{\circledR}$ epoxy quick set glue, and the drying is taking place from the other extremity. With hydrophilic surface, filling the tube with a solution is readily obtained by putting one of its extremity in contact with the liquid, which, due to capillary effects, penetrates and fills the tube. With hydrophobic surface it is necessary to inject the liquid with a syringe from one of the tube extremities. Then the open tube end is exposed to a stagnant air atmosphere at ambient temperature $\left(25^{\circ} \mathrm{C}, 50 \% \mathrm{RH}\right)$, and evaporation starts. With a Carl Zeiss Microscopy ${ }^{\circledR}$ device we follow the drying process by taking successive top view images. 


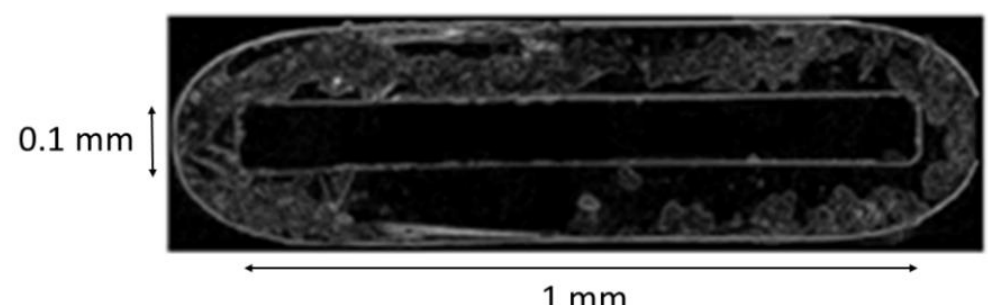

Figure 1: Cross-section of the tube.

When conducting test with hydrophilic tubes, the interior of the capillary tube is cleaned with nitric acid at $50 \%$ then rinsed with pure water. This allows to get a hydrophilic surface, with a $0^{\circ}$ contact angle with pure water (about $9^{\circ}$ with the sulfate calcium solution). When conducting tests with hydrophobic tubes, the surfaces were prepared differently by placing a silane solution (obtained by mixing $0.01 \mathrm{~g}$ of trichlorosilane with $10 \mathrm{mg}$ of octane) inside the tube during one day, which induces a solvent-based salinization reaction (Brzoska et al. 1994). The solution is then removed and the tube is dried. This allows us to get a hydrophobic surface, with a $120^{\circ}$ contact angle with pure water. The contact angles were measured using the goniometer method; a droplet is deposited by a syringe on the surface, and a high resolution camera captures the image from the side view, then the angle is determined using an image analysis software.

Different liquid solutions were used. We used pure (distilled) water. The ionic solution was basically a calcium - sulfate saturated water solution made by placing during few hours $100 \mathrm{~g}$ of gypsum powder in $1 \mathrm{I}$ of water so as to obtain a saturated solution (i.e. $2.65 \mathrm{~g} / \mathrm{l}$ ). Note that this rather low solubility can be at the origin of physical trends during drying rather different from those observed with salt solutions with much larger solubility such as $\mathrm{NaCl}$. Then various other materials were used to study the impact of different effects such as the wetting characteristics of the solution, the crystallisation characteristics of the ions, or the size of the suspended elements (colloids) in the liquid. A different ionic solution was obtained by adding citric acid (at a concentration of $10 \mathrm{~g} / \mathrm{l}$ ) in calcium - sulphate saturated solution. At last, we also changed the wettability of the ionic solution by adding surfactants. In order to avoid any interplay between these surfactants and the crystallization phenomenon, we used non-ionic surfactants, i.e. PEG $\left(\left(\mathrm{C}_{3} \mathrm{H}_{6} \mathrm{O} . \mathrm{C}_{2} \mathrm{H}_{4} \mathrm{O}\right)_{x}\right.$, number average molecular weight $\left.\mathrm{Mn} \approx 4400\right)$ and Sorbitan esters (Tween 20). They were mixed in a gypsum-saturated solution so as to obtain a 3.3 $\mathrm{g} / \mathrm{l}$ concentration. The contact angles of the ionic solutions mixed with these surfactants on hydrophilic surfaces is $4-5^{\circ}$.

The order of magnitude of the velocity of liquid transport $(U)$ during our tests is a channel length $L=2.5 \mathrm{~cm}$ emptied in $200 \mathrm{~min}$, which gives $2 \times 10^{-6} \mathrm{~m} . \mathrm{s}^{-1}$, so that we have $U L=5 \times 10^{-8} \mathrm{~m} . \mathrm{s}^{-1}$, a value much larger than the coefficient of diffusion of any species in water (ions, colloids), smaller than the self-diffusion coefficient of water $\left(D=2.3 \times 10^{-9} \mathrm{~m}^{2} \cdot \mathrm{s}^{-1}\right)$. Note that in some cases the velocity drops to a value about five times smaller but this does not change the conclusion. Thus the Peclet number (i.e. $P e=U L / D)$ is much larger than 1 in all our tests, so that we expect that advection is dominant.

\section{Results and discussion}

During drying of the capillary with a hydrophilic surface filled with water we observe that the air-liquid interface deforms in time. Initially it is perpendicular to the tube axis (see Fig.2a i)), then it progressively curves towards the tube interior (see Fig.2a ii) and iii)). At some time it becomes composed of two interfaces along the sides in the tube direction and one interface with a large radius of curvature at 
the front (see Fig.2a iv) and v)). This "steady-state" shape remains the same until the end of drying, while the front progressively penetrates deeper in the tube.

(a)

i)

ii)

iii)
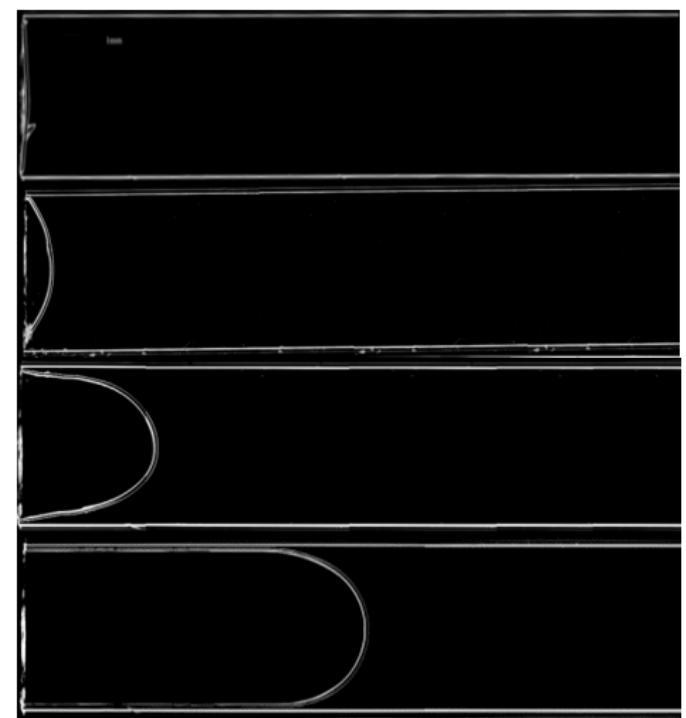

iv)

v)

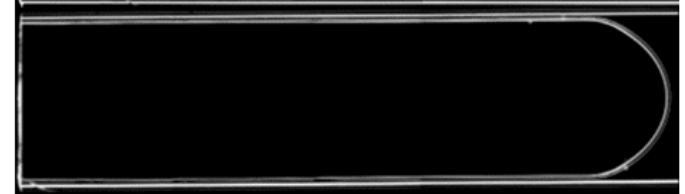

(b)

i)

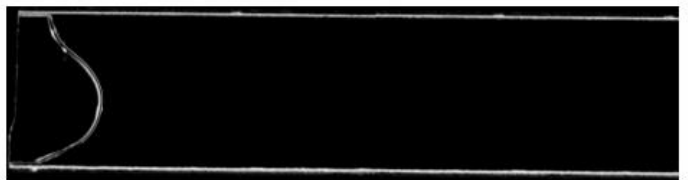

ii)

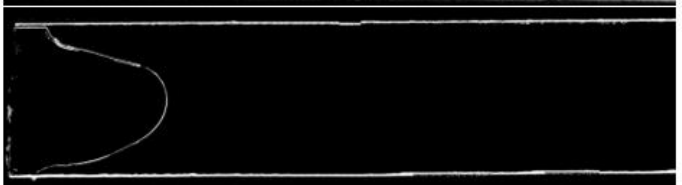

iii)

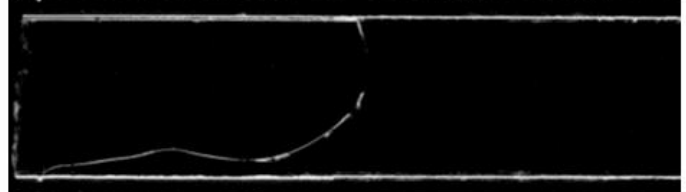

iv)

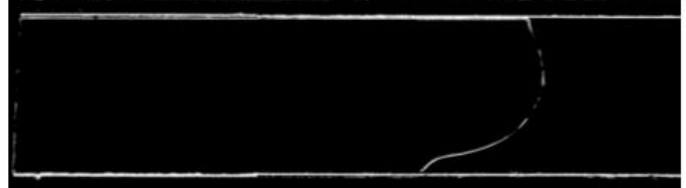

v)

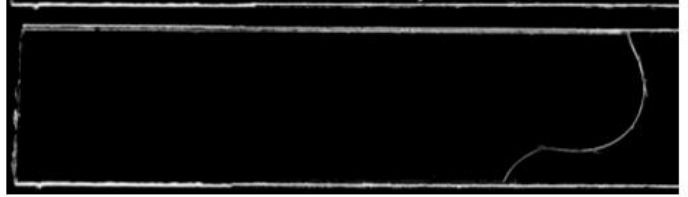

Figure 2: Views of the liquid air interface in the main plane of the channel at different times for pure water and hydrophilic surface (a): i) 0 , ii) 0.6 , iii) 14 , iv) 45 , v) $80 \mathrm{~min}$; or hydrophobic surface (b): i) 6, ii) 33, iii) 45, iv) 89, v) $186 \mathrm{~min}$. (open side: left hand side)

With this tube shape, which has a cross-section close to a perfect rectangle, the interface along the tube sides is so close to the corner that it is not possible to get precise values of the radius of curvature in a plane perpendicular to the main plane. However the physical processes at work are similar to those mentioned in Keita and al. 2016 a): the Laplace pressure is at any time constant along all the interface, which means that the evolution of the interface shape is governed by capillary effects.

We can estimate the liquid volume loss from the apparent interface evolution while neglecting the curvature in the plane perpendicular to the main plane. This is done by computing for each picture the volume left free behind the apparent interface in the main plane. Then we represent the saturation, which we here define as the ratio of liquid volume still present in the tube to the initial liquid volume, as a function of time. It appears that the saturation decreases linearly in time (see Figure 3) over our typical range of observations, i.e. a front penetration depth of about $25 \mathrm{~mm}$.

Such a result is explained by the same arguments as in Keita et al. (2016 a): the air-liquid interface is pinned at the tube entrance, which imposes the existence of liquid films from the main front (inside the tube) to the tube entrance; the presence of these liquid-air interfaces in a confined system maintains a vapor density close to 1 in the air, which damps the evaporation; as a consequence, evaporation mainly occurs from the liquid-air interface around the tube entrance; since its shape remains apparently constant in this region, the evaporation remains constant; as a corollary the water transport inside the tube essentially takes place in the form of liquid moving in the thin films along the wall, which drains liquid from the large volume behind the main front, which can thus progress inwards. 
Following the computation of Keita et al. 2016a that the relative humidity $(n)$ is in that case close to 1 around the channel exit, we can roughly consider that the evaporation is due to vapor diffusion through a layer of constant thickness $\delta$ outside the channel. Under such conditions, from the second Fick's law, we get the water mass evaporating by unit time: $d m_{w} / d t=-\rho_{0} S D_{0} \nabla n=-\rho_{0} S D_{0} / \delta$, with $S$ the sample cross-section, $D_{0}=2.7 \times 10^{-5} \mathrm{~m}^{2} . \mathrm{s}^{-1}$ the coefficient of diffusion of vapor in the air, and $\rho_{0}=23.4 \mathrm{~g} . \mathrm{m}^{-3}$ the maximum vapor density in air. In the case of hydrophilic surface (i.e. data in Fig.3) we deduce that it is necessary to use $\delta \approx 0.3 \mathrm{~mm}$ in the above equation to find the observed drying rate.

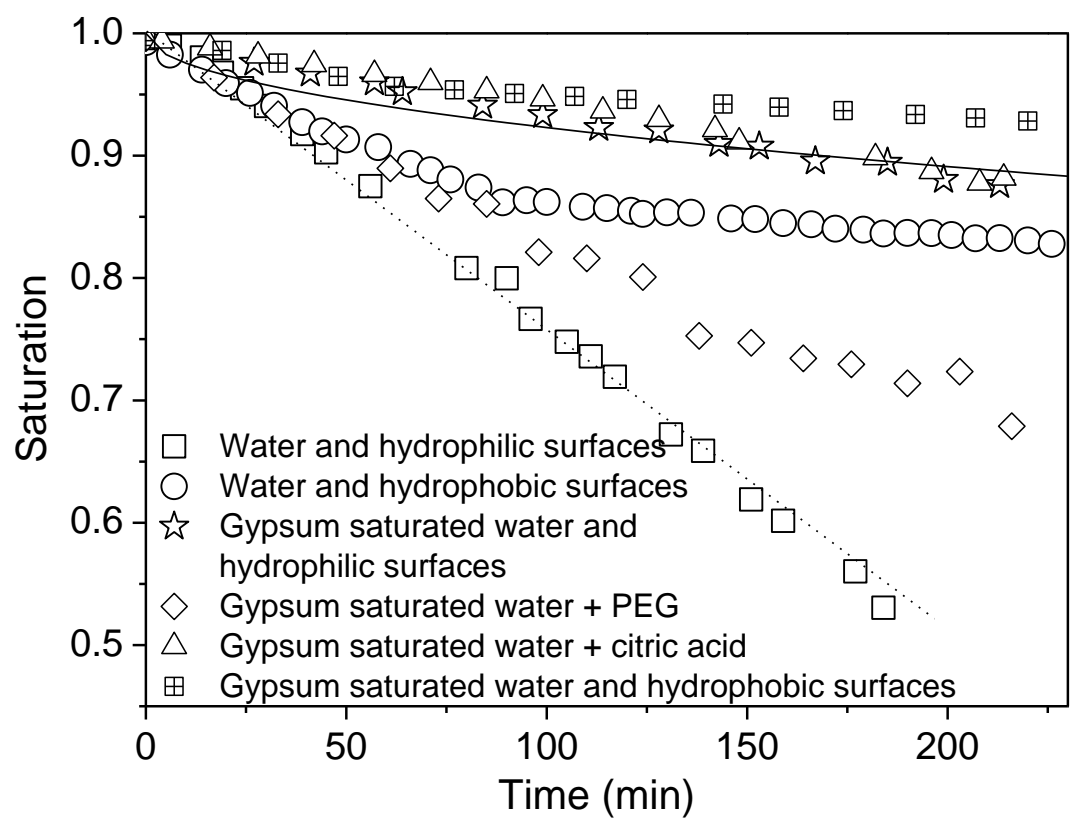

Figure 3: Saturation as a function of time for the tests shown in Figures $2 a$ and $2 b$, i.e. distilled water with hydrophilic surface (squares) and hydrophobic surface (circles). The stars show data for gypsum saturated water with hydrophilic surface. The diamonds show data for gypsum saturated water plus PEG with hydrophilic surface. The triangles show data for gypsum saturated water + citric acid. Cross-squares saturated gypsum water with hydrophobic surface. The dotted line is a guide for the eyes. The continuous line is the prediction of the diffusion model assuming perfect dewetting (see text).

With hydrophobic surface tubes the evolution of the liquid-air interface during drying is completely different. We have a first stage during which this interface strongly deforms while remaining pinned at least on one side close to the entrance (see Fig. $2 \mathrm{~b}$ i) and ii)). Then come a second stage when the interface is no longer pinned, and progresses inwards while keeping a roughly constant shape (see Fig. $2 \mathrm{~b}$ iv) and $\mathrm{v}$ )). There may also exist a transient regime for which only one side is unpinned (see Fig. $2 \mathrm{~b}$ iii)). Note that there are no liquid films ahead of the points of contact of the main interface and the channel sides. This is consistent with the shape of the interface reaching the wall: the apparent slope discontinuity in the main plane reflects a separation between a wet and a liquid region, which should exhibit a large contact angle due to the hydrophobic nature of the solid surface.

Because of the randomness feature of the crystallization phenomenon, it is important to keep in mind that the results are reproducible from a qualitative point of view, i.e. the two regimes and the 
corresponding trends are obtained in each case, but the exact shape of the interface and the time for unpinning vary from one test to another. In this context we can only discuss global tendencies with such data. We observe a decrease of the drying rate when the saturation decreases (see Fig.3) and due to the fact that the interface unpins from one or two sides there is no more liquid film allowing a fast transport towards the channel entrance. Instead, drying now relies on evaporation from a certain depth to the channel entrance, then vapor has to diffuse across this depth to reach the exit.

Finally the two different processes observed above for drying in a single channel with the two types of surfaces are quite analogous to the two different processes observed for drying of a model porous medium, i.e. a granular packing: with also such two types of surfaces (hydrophilic or hydrophobic) this leads respectively to drying at a constant rate or to a continuously decreasing rate of drying from the beginning of the test (Shahidzadeh-Bonn et al 2007).

\section{Impact of the presence of elements in suspension in the liquid}

In this preliminary study of the flow characteristics during drying of a channel filled with a pure liquid, and before considering the case of a saline solution which is the main scope of this work, it seems useful to have a look at the impact of the presence of some "coarse" elements in suspension in the liquid. Here we consider the case of stabilized hydrophilic colloidal silica nanoparticles (Ludox HS-40 diameter: $20 \mathrm{~nm}$, density 2.45) suspended in water $(\mathrm{pH}=9.8$ to maintain the particle electrostatic repulsion), so that particle segregation or sedimentation are negligible. The experiment is carried out in the channel type used in Keita et al. (2016 a), which gives liquid films of larger thickness along the channel sides, when the main interface progresses inwards. Here, with a suspension, we also see initially a wide interface forming around the channel entrance and growing inward (see Fig.4, 0 and 11 min), as for a pure liquid (see Keita et al. 2016 a) for the same channel shape or Fig.2a for our standard channel shape). However we also observe that the liquid films along the channel side soon transform in regions of particle accumulation which roughly appear as "crystallized" areas with fractures (see Fig.4, 67, 149, $186 \mathrm{~min}$ ). These fractures indicate the presence of a compacted zone, similar to previous observation in a channel (Dufresne et al. 2003) or in porous media (Keita et al. 2014, Keita et al. 2016 b). These areas grow in time as the main interface progresses farther. Finally, when the main interface reaches the channel extremity, the drying process is now taking placing via evaporation of the side films whose thickness decreases. Also, the "crystallized" area goes on growing but its thickness progressively decreases (see Fig.4, 210, 229, $290 \mathrm{~min}$ ). 


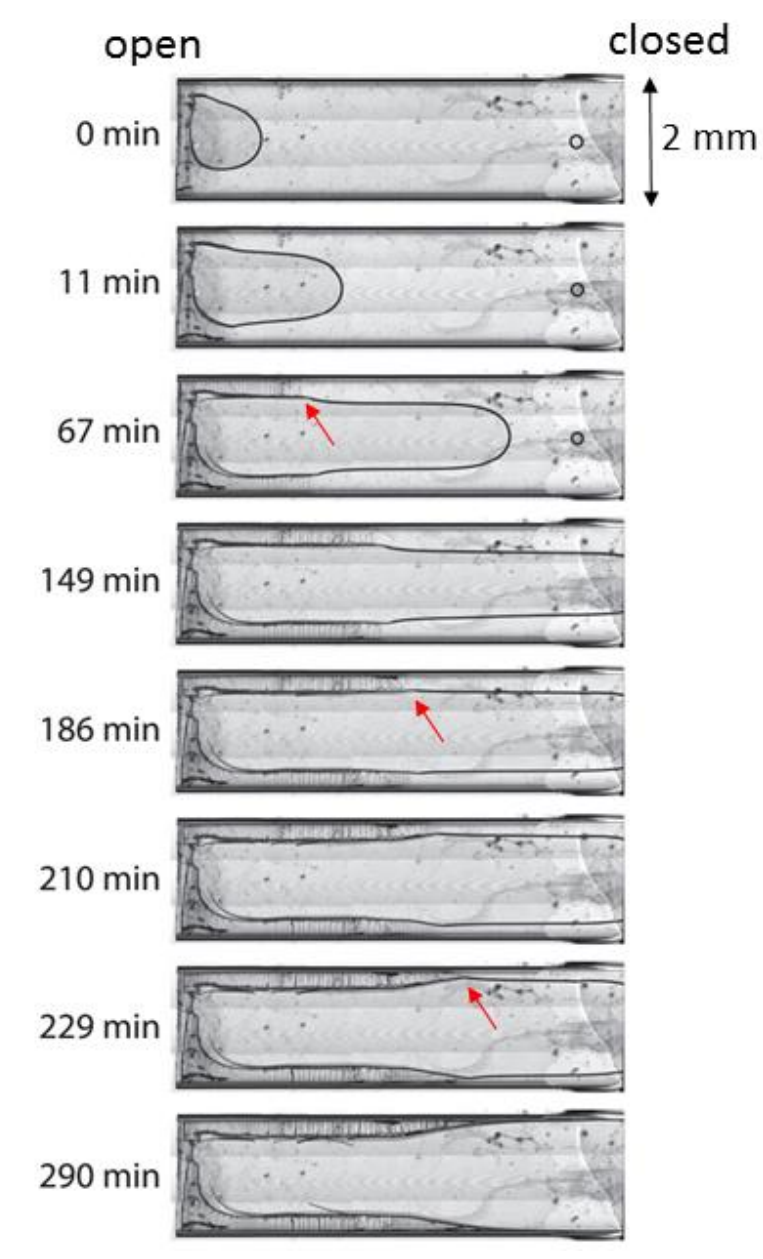

Figure 4: Successive views (at different times) of the drying of a Ludox suspension (10\%) in a capillary (open side: left hand). The growing curved line corresponds to the liquid airinterface position (see text). The region of particle deposit is the darker grey region along the (upper and lower) walls. The region extends over an increasing distance inside the channel, which corresponds to the front position shown by red arrows in some pictures.

These original drying characteristics have a rather simple qualitative explanation. Considering the Peclet number value, the suspended particles, although colloidal, do not have time to diffuse significantly through the liquid, they are essentially transported with it. Since we have seen that most of the evaporation takes place around the extremity of the side films where the liquid is transported from the interior of the channel, the suspended particles follow this motion and stop at the liquid-air interface at this film extremity. The accumulated particles then can tend to dry or remain somewhat wet if evaporation from this medium (particles and water) is too slow, but anyway we cannot expect a significant liquid motion through the porous medium formed by this particle packing whose permeability is extremely small (it scales with the square of the particle radius). Assuming such flow is negligible we deduce that evaporation will now take place essentially around the front of the crystallized area progressing inwards. This explains the continuous decrease of the drying rate in time (see Fig.5), as drying relies on the evaporation then vapor diffusion across this forming (through particles accumulation) zone. Finally, when the main interface has reached the channel end and the side film thickness decreases, the amount of particles, associated with an elementary liquid evaporation and further advance of the particles deposit region, decreases, which explains that the deposit thickness decreases. Actually this scheme corresponds to that assumed to occur for the drying 
of the same type of suspension in a porous medium, which was finally illustrated by a schematic description for a single channel representing the porous medium (see Keita et al. 2014).

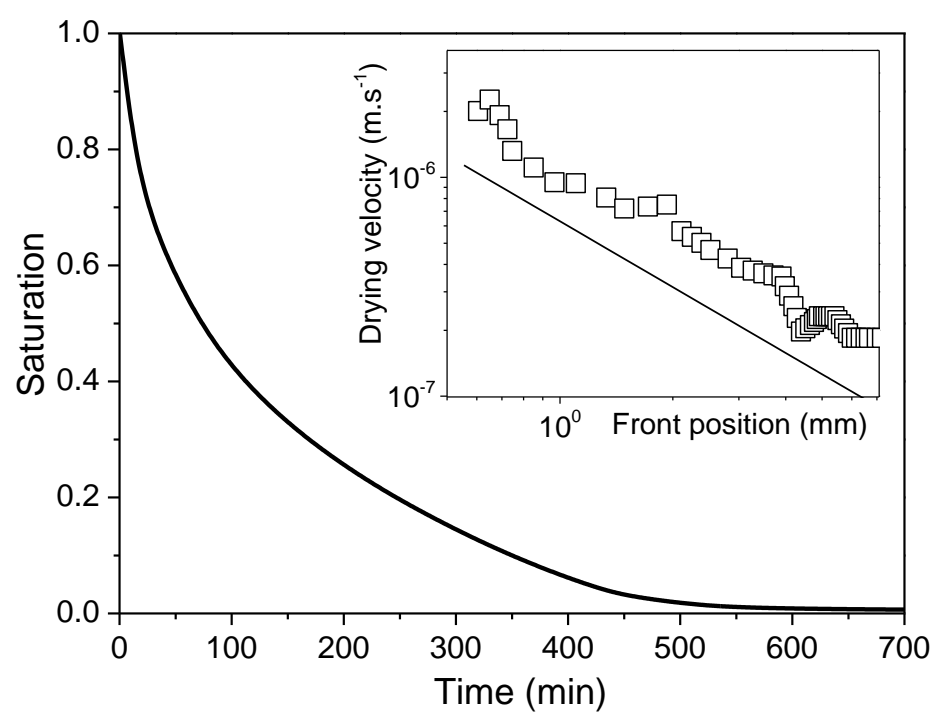

Figure 5: Saturation vs time for the drying of a channel filled with a Ludox suspension (same test as in Figure 4). The inset shows the drying rate $(V)$ as a function of the front position. The continuous line is the theoretical drying rate assuming evaporation via vapor diffusion in the channel from a vapor saturated region at the front position (see text).

Let us assume that a dry region develops inside the sample from its exit. In that case we can assume that the liquid evaporates exactly from the limit of the wet region, where $n=1$, and the vapor diffuses to the free surface over a distance $h$, i.e. the thickness of the dry region, before reaching the external boundary layer. Note that the saturated front occupies the whole cross-section, so that diffusion is essentially 1D (along the main channel direction). As soon as $h$ is larger than a few millimeters we have $h \gg>\delta$ so that we can neglect the diffusion process outside the channel, the diffusion process inside the channel being dominant. Under such conditions, from the above expression of the Fick's law, the drying rate, defined as $V=-(1 / S \rho) d m_{e} / d t$, in which $\rho$ is the liquid density, expresses as $V=\rho_{0} D_{0} / \rho h$. The corresponding drying rate values, considering that $h$ is given by the front position determined from the pictures (see Fig.4), appear to be smaller than the observed values on average by a factor about 2 . Thus the assumption of vapor diffusion mainly starting from the front position is apparently wrong. Some significant evaporation would rather take place from other points along the particle deposit, which would accelerate drying with regards to the above model prediction. As a corollary, some liquid flow should be maintained through the particle deposit region, which would thus not be perfectly dry.

\section{Drying of an ionic solution}

When drying an ionic solution in a hydrophilic capillary, we at first sight get the same behavior as without ions in solution: the interface starts to deform and when it has reached some specific shape it progresses inwards, leaving a liquid film behind it along the channel sides (see Fig.6 left). However we 
can also see that deposits of crystals are left behind the main interface along the sides (see Fig.6b). These deposits grow in time, in thickness and in depth along the channel axis inwards (see Fig.6b,c,d). This result is somewhat unexpected: if the liquid film was still pinned at the channel entrance as for the drying of pure water (see Figure 2) we would expect a progressive accumulation of crystals at more or less the same place close to the entrance. Here it appears that some crystal deposit may be formed along the channel side at some time (see Fig.7a), and then stops growing (see the main crystal on the left in Fig.7b and next ones). At the same time a new crystal deposit is formed farther inward and significantly grows (see main deposit on the right in Figure 7). This phenomenon appears to be associated with a kind of dewetting of the wall between the two deposits: in Figure 7 we can see that the main liquid film (in the foreground) beyond the first deposit along the channel side (shown by the yellow arrow in Figure 7a), disappears between the first and second picture, while simultaneously the next deposit appears, so that afterwards one may see a liquid film (shown by a red arrow in Figure 7b) in the forefront only beyond this new farthest deposit. The newly formed deposit will then grow as long as the liquid film remains pinned to it (see Figure 7c,d,e). Actually a side view (see Fig.7f) also shows that there is no discontinuity of the crystal deposit along the wall, which means that the film likely dewetted the wall progressively. Therefore, there is a dewetting effect linked to the crystallization process.

a)

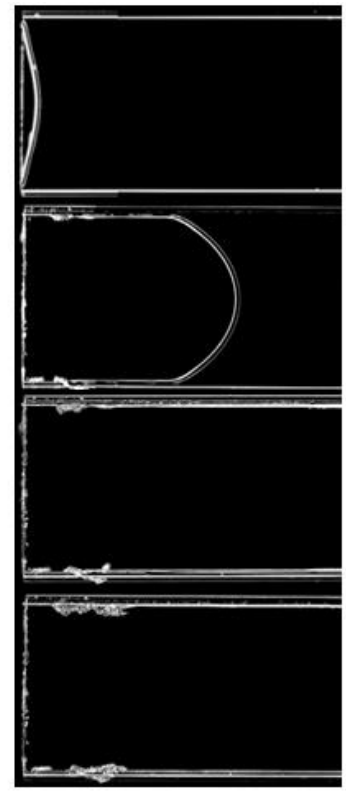

$\left.a^{\prime}\right)$

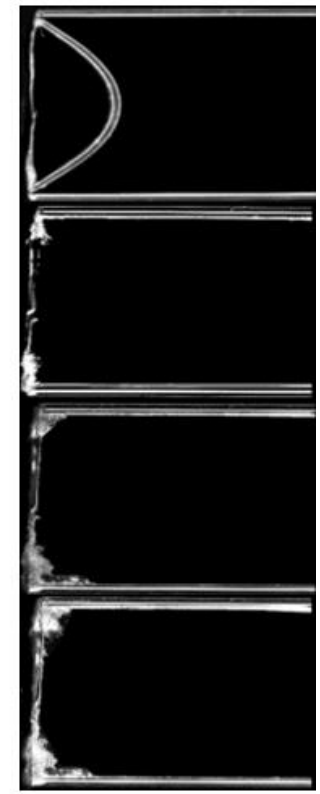

Figure 6: (left) Successive views of a Calcium sulfate solution drying in a hydrophilic capillary at times : a) 0, b) 27 min., c) 57 min., d) 153 min. (right) Successive views of a Calcium sulfate solution with tween 20 drying in a hydrophilic capillary at times : a') 2 min., b') 17 min., c') 56 min., d') 94 min. Note that after its initial visible evolution on the first pictures, the main liquid-air interface further progresses on the right and is not visible on the next pictures. 


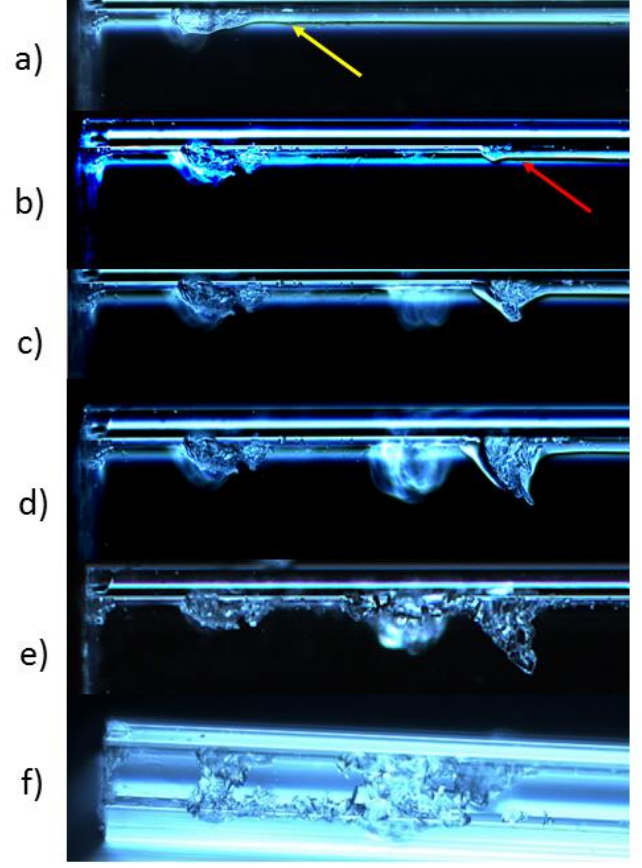

Figure 7: Successive views of the drying of the drying of a Calcium sulfate solution in a hydrophilic capillary: zoom on one of the wall. The arrow meaning is explained in the text.

According to these observations we deduce that the formation of crystal deposit tends to induce some depinning of the line of contact, which then progressively moves inwards with the crystal deposit. A similar effect was inferred from observations (with MRI) of drying characteristics of plasters (Seck et al. 2016). This effect induces a decrease of the drying rate, since the line of contact, around which most evaporation occurs, moves away from the channel entrance, so that vapor has to diffuse through a growing distance.

For the drying of an ionic solution in a channel with hydrophobic surface the same phenomena as for pure water (see Fig.2b) is observed: a main interface without liquid film along the wall sides and with a somewhat chaotic evolution, but on average essentially a drying associated with a dewetting of the channel from its entrance inwards. This has a particular consequence for the formation of crystals: as there is no particular path of liquid transport in the channel, there is no particular region of accumulation and instead the deposits are approximately uniformly distributed along the different walls (see Fig.8). This reflects a rather regular withdrawing of the main interface in time, so that the drying rate continuously decreases (see Fig.3) as the front of evaporation withdraws in the capillary. Finally the drying rate is smaller than that observed for hydrophilic surfaces (see Fig.3), simply because with hydrophobic surfaces the front withdrawal is maximized, i.e. the evaporation is directly associated with front withdrawal.

In that case it is interesting to compare the prediction of the above diffusion model with data. The drying rate, i.e. $V$, may also be written $d h / d t$, and after integration we get $h=\sqrt{2 \rho_{0} D_{0} t / \rho}$. The saturation then evolves as $\phi=1-h / L_{0}$, in which $L_{0}$ is the full channel length. This theoretical saturation evolution compares relatively well with data with gypsum saturated solution (without surfactants), for which a more or less progressive dewetting was observed (see Fig.3). However, the predicted drying rate is somewhat larger that the observed one in the ideal case of hydrophobic 
surface which corresponds exactly to the model assumptions, an effect for which we have no explanation.

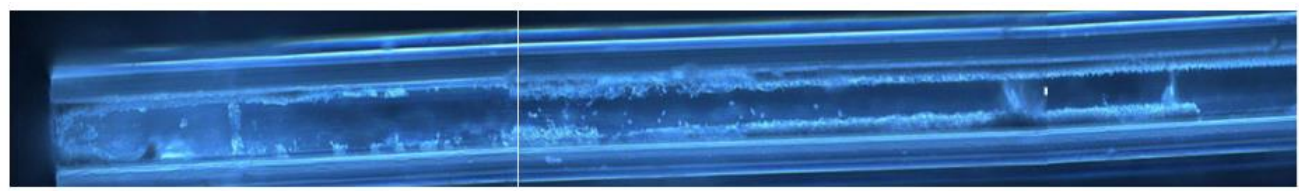

Figure 8: Side view of the hydrophobic capillary after drying of a Calcium sulfate solution.

Adding citric acid in a gypsum solution generally induces a delayed crystallization as it is one of the most efficient settling retarder used for gypsum (Singh and Middendorf 2007), which affects crystal morphology. Using this type of additive we get a rate of drying rather close to that for a simple saturated gypsum solution (see Fig.3). The aspect of crystals is nevertheless effectively different but they are still formed all along the wall of the channel (see Fig.9), indicating a priori a progressive withdrawal of the wetted region. This is the marker of a limited liquid transport in the region of crystal deposits, but it is not possible to conclude that there are not still some slight residual flow and evaporation in this region.

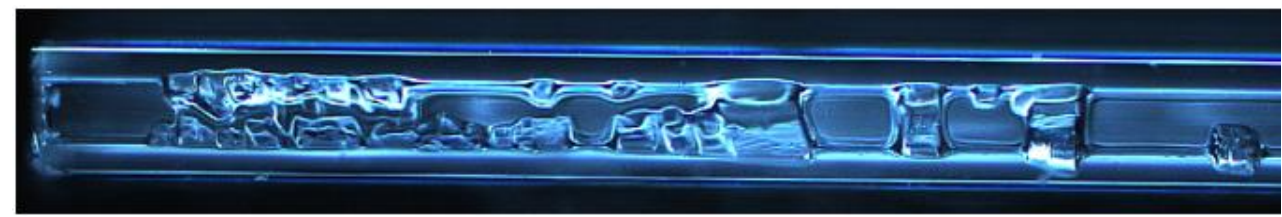

Figure 9: Side view of the hydrophilic capillary after drying of a Calcium sulfate solution plus citric acid.

Finally, we can further test the impact of wetting properties of the ionic solution by adding surfactant in a gypsum saturated solution. Then, we can see a significant acceleration of the drying process (see Fig.3), which now occurs at a rate not too far from that observed for a pure water solution. Note that the impact of surfactants on the evaporation rate of $\mathrm{NaCl}$ solutions was observed to be negligible (Qasi et al. 2017), so that assuming this is the same for the Calcium sulfates solutions our observations imply that the drying rate essentially depends on the liquid distribution in the capillary. Moreover, the surfactants may, in some cases, be incorporated in the crystals (Qasi et al. 2017), but we will not particularly study this aspect as we have chosen non-ionic surfactants to discard this possibility. Looking at the evolution of the crystal distribution in time we find that, in contrast with observations for the gypsum saturated solutions (see Figs.6-9), crystals develop essentially and accumulate just at the channel entrance (see Fig.6right). This means that, all along the process, the liquid now wets the channel up to a distance close to the entrance, and thus can continuously feed ions in this region. Thus we have a continuous liquid film along the wall, which allows a liquid transport towards the channel entrance where it evaporates. This explains why the drying rate remains close to that observed for pure water in a hydrophilic channel (see Fig.3). Thus the presence of surfactants allows to avoid the 


\section{Conclusion}

In the objective of understanding the impact of crystallization on the drying rate of porous media filled with low solubility ionic solutions, we carried out tests with an extremely simple structure, namely a thin straight channel, in which capillary effects are dominant throughout the process of liquid extraction. From a general point of view, it appears that the impact on the drying rate will essentially depend on the position of the line of contact induced by such effects, resulting in a decrease of the drying rate if the line of contact is progressively displaced inwards. Even in such a simple geometry we observe a great sensitivity of the drying rate to the elements initially suspended in the liquid and which can deposit along the wall. This is due to the fact that the drying rate results from the gradient of vapor density in the channel, which depends on the liquid distribution along the channel walls, which in turn is very sensitive to the formation of deposits or to dewetting processes associated to the crystallization. This implies that small changes in the formulation of the solution or the wetting characteristics of the porous medium may strongly affect the drying rate. These findings could be directly applied to the manufacturing of building materials by decreasing their drying time through just an optimization of their formulation, which will generate huge cost savings in terms of energy.

\section{References}

Bergstad, M., Shokri, N., Evaporation of $\mathrm{NaCl}$ from porous media with mixed wettability, Geophys.

Res. Lett., 43, 4426-4432 (2016)

Brzoska, J.B., Ben Azouz, I., Rondelez, F. Silanization of solid substrates: a step toward reproductibility, Langmuir, 10, 4367-4373 (1994)

Camassel, B., Sghaier, N., Prat, M., Ben Nasrallah, S. Evaporation in a capillary tube of square crosssection: application to ion transport, Chemical Engineering Science, 60, 815-826 (2005)

Derluyn, H., Griffa, M., Mannes, D., Jerjen, I., Dewanckele, J., Vontobel, P., Sheppard, A., Derome, D., Cnudde, V., Lehmann, E., Carmeliet, J. Characterizing saline uptake and salt distributions in porous limestone with neutron radiography and X-ray micro-tomography. J. Building Physics, 36, 353-374 (2013)

Desarnaud, J., Bertrand, F., Shahidzadeh-Bonn, N. Impact of the kinetics of salt crystallization on stone damage during rewetting/drying and humidity cycling. J. Appl. Mech., 80, 020911 (2013)

Desarnaud, J., Derluyn, H., Molari, L., de Miranda, S., Cnudde, V., Shahidzadeh, N. Drying of salt contaminated porous media: Effect of primary and secondary nucleation. J. Appl. Phys., 118, 114901 (2015)

Dufresne, E.R., Corwin, E.I., Greenblatt, N.A., Ashmore, J., Wang, D.Y., Dinsmore, A.D., Cheng, J.X., Xie, X.S., Hutchinson, J.W., and Weitz, D.A. Flow and Fracture in Drying Nanoparticle Suspensions, Phys. Rev. Lett., 91, 224501 (2003)

Eloukabi, H., Sghaier, N., Prat, M., Ben Nassrallah, S. Drying Experiments in a Hydrophobic Model Porous Medium in the Presence of a Dissolved Salt, Chem. Eng. Technol., 34, 1085-1094 (2011)

Espinosa-Marzal, R.M., Scherer, G.W. Advances in Understanding Damage by Salt Crystallization. Accounts of Chemical Research, 43, 897-905 (2010)

Espinosa-Marzal, R.M., Scherer, G.W. Impact of in-pore salt crystallization on transport properties. Environ. Earth Sci., 69, 2657-2669 (2013) 
Flatt, R.J. Salt damage in porous materials: how high supersaturations are generated. J. Crystal Growth., 242, 435-454 (2002)

Flatt, R.J., Caruso, F., Aguilar Sanchez, A.M., Scherer, G.W. Chemo-mechanics of salt damage in stone. Nature Communications, 5, 4823 (2014)

Foraboschi, P., Vanin, A. Experimental investigation on bricks from historical Venetian buildings subjected to moisture and salt crystallization. Eng. Failure Analysis. 45, 185-203 (2014)

Gupta, S., Huinink, H.P., Pel, L., Kopinga, K. How ferrocyanide influences $\mathrm{NaCl}$ crystallization under different humidity conditions. Crystal Growth Design, 14, 1591-1599 (2014 a)

Gupta, S., Huinink, H.P., Prat, M., Pel, L., Kopinga, K. Paradoxical drying of a fired-clay brick due to salt crystallization. Chem. Eng. Sci., 109, 204-211 (2014 b)

Hidri, F., Sghaier, N., Eloukabi, H., Prat, M., Ben Nasrallah, S., Porous medium coffee ring effect and other factors affecting the first crystallization time of sodium chloride at the surface of a drying porous medium, Physics of Fluids, 25, 1-23 (2013)

Keita, E., Faure, P., Rodts, S., Coussot, P., MRI evidence for a receding-front effect in drying porous media. Phys. Rev. E., 87, 062303 (2014)

Keita, E., Kodger, T.E., Faure, P., Rodts, S., Weitz, D.A., Coussot, P. Water retention against drying with soft-particle suspensions in porous media, Phys. Rev. E, 94, 33104 (2016 b)

Keita, E., Koehler, S.A., Faure, P., Weitz, D.A., Coussot, P. Drying kinetics driven by the shape of the air/water interface in a capillary channel, Eur. Phys. J. E, 39, 23 (2016 a)

Kramar, S., Urosevic, M., Pristacz, H., Mirtic, B., Assessment of limestone deterioration due to salt formation by micro-Raman spectroscopy: application to architectural heritage. J Raman Spectrosc, 41, 1441-1448 (2010)

Liu, Z., Deng, D., De Schutter, G. The damage of calcium sulfoaluminate cement paste partially immersed in MgSO4 solution. Constr and build mater. 66, 692-711 (2014)

Naillon, A., Duru, P., Marcoux, M., Prat, M. Evaporation with sodium chloride crystallization in a capillary tube, Journal of Crystal Growth, 422, 52-61 (2015)

Norouzi Rad, M., Shokri, N., Nonlinear effects of salt concentrations on evaporation from porous media, Geophys. Res. Lett, 39, L04403 (2012)

Norouzi Rad, M., Shokri, N., Kesmiri, A., Withers, P.J. Effects of grain and pore size on salt precipitation during evaporation from porous media: A pore-scale investigation. Transp. Porous Med., 110, 281-294 (2015)

Pel, L., Huinink, H., Kopinga, K., van Hees, R.P.J., Adan, O.C.G. Efflorescence pathway diagram: understanding salt weathering. Constr Build Mater., 18, 309-313 (2004)

Petkovic, J., Huinink, H.P., Pel, L., Kopinga, K., van Hees, R.P.J. Moisture and salt transport in threelayer plaster/substrate systems. Constr Build Mater., 24, 118-127 (2010)

Petkovic, J., Huinink, H.P., Pel, L., Kopinga, K., van Hees, R.P.J., Salt transport in plaster/substrate layers. Mat and Struct., 40, 475-490 (2007)

Peysson, Y., Bazin, B., Magnier, C., Kohler, E., Youssef, S. Permeability alteration due to salt precipitation driven by drying in the context of $\mathrm{CO} 2$ injection. Energy Proc. 4, 4387-4394 (2011)

Qazi, M.J., Liefferink, R.W., Schlegel, S.J., Backus, E.H.G., Bonn, D., Shahidzadeh, N. Influence of Surfactants on Sodium Chloride Crystallization in Confinement, Langmuir 33, 4260-4268 (2017)

Rodriguez-Navarro, C., Doehne, E. Salt Weathering: Influence of Evaporation Rate, Supersaturation and Crystallization Pattern. Earth Surf. Process. Landforms. 24, 191-209 (1999)

Rufai, A., Crawshaw, J., Micromodel observations of evaporative drying and salt deposition in porous media, Physics of Fluids 29, 126603 (2017) 
Sawdy, A., Heritage, A., Pel, L. A review of salt transport in porous media, assessment methods and salt reduction treatments. In Proceedings International Conference on Salt Weathering on Buildings and Stone Sculptures, Technical University of Denmark. Lyngby 1-28. (2008)

Scherer, G.W. Stress from crystallization of salt. Cement Concrete Res., 34, 1613-1624 (2004)

Schiro, M., Ruiz-Agudo, E., Rodriguez-Navarro, C. Damage Mechanisms of Porous Materials due to InPore Salt Crystallization. Phys. Rev. Lett., 109, 265503 (2012)

Schultz, P., Schlünder, E.U. Influence of additives on crust formation during drying. Chem. Eng. Process, 28, 133-142 (1990)

Seck, M.D., Keita, E., Faure, P., Cavalié, P., Van Landeghem, M., Rodts, S., Coussot, P. Subflorescence and plaster drying dynamics, Chem. Eng. Sci., 148, 203-211 (2016)

Seck, M.D., Understanding drying mechanisms in building materials: the case of plaster, PhD thesis, Univ. Paris-Est, 2015 (in French)

Seck, M.D., Van Landeghem, M., Faure, P., Rodts, S., Combes, R., Cavalié, P., Coussot, P. The mechanisms of plaster drying. J. Mat. Sci., 50, 2491-2501 (2015)

Sghaier, N., Prat, M. Effect of efflorescence formation on drying kinetics of porous media. Transp.

Porous Med., 80, 441-454 (2009)

Shahidzadeh-Bonn, N., Azouni, A., Coussot, P. Drying rate controlled by wettability in model porous media, Journal of Physics: Condensed Matter, 19, 112101 (2007)

Shahidzadeh-Bonn, N., Desarnaud, J., Bertrand, F., Chateau, X., Bonn, D., Damage in porous media du to salt crystallization, Phys. Rev. E., 81, 066110 (2010)

Shahidzadeh-Bonn, N., Rafaï, S., Bonn, D., Wegdam, G. Salt crystallization during evaporation: impact of interfacial properties. Langmuir, 24, 8599-8605 (2008)

Shokri-Kuehni, S.M.S., Vetter, T., Webb, C., Shokri, N., New insights into saline water evaporation from porous media: complex interaction between evaporation rates, precipitation, and surface temperature, Geophys. Res. Lett., 44, 5504-5510 (2017)

Singh, N.B., Middendorf, B., Calcium sulphate hemihydrate hydration leading to gypsum crystallization. Progress in Crystal Growth and Characterization of Materials, 53, 57-77 (2007)

Tsui, N., Flatt, R.J., Scherer, G.W. Crystallization damage by sodium sulfate. J. Cultural Heritage, 4, 109-115 (2003)

Veran-Tissoires, S., Marcoux, M., Prat, M. Discrete Salt Crystallization at the Surface of a Porous Medium. Phys. Rev. Lett., 108, 054502 (2012)

Vosten, B., Hersfeld, B. The drying of crust-forming materials as exemplified by the drying of gypsum wallboard (in German). Zement-Kalk-Gips., 5, 213-222 (1976) 